\title{
A New Class of Wolf-Rayet Stars: WN3/O3s
}

\author{
Philip Massey ${ }^{1}$, Kathryn F. Neugent ${ }^{1}$, Nidia Morrell $^{2}$ and \\ D. John Hillier ${ }^{3}$ \\ ${ }^{1}$ Lowell Observatory \\ email: phil.massey@lowell.edu \\ ${ }^{2}$ Las Campanas Observatory, Carnegie Observatories \\ ${ }^{3}$ Department of Physics and Astronomy \& Pittsburgh Particle Physics, Astrophysics, and \\ Cosmology Center, University of Pittsburgh
}

\begin{abstract}
Our new survey for Wolf-Rayet stars in the Magellanic Clouds is only $15 \%$ complete but has already found 9 new Wolf-Rayet (WR) stars in the Large Magellanic Cloud (LMC). This suggests that the total WR population in the LMC may be underestimated by $10-40 \%$. Eight of the nine are of the WN subtype, demonstrating that the "observed" WC to WN ratio is too large, and is biased towards WC stars. The ninth is another rare WO star, the second we have found in the LMC in the past two years. Five (and possibly six) of the 8 WNs are of a new class of WRs, which pose a significant challenge to our understanding. Naively we would classify these stars as "WN3+O3V," but there are several reasons why such a pairing is unlikely, not the least of which is that the absolute visual magnitudes of these stars are faint, with $M_{V} \sim-2.3$ to -3.1 . We have performed a preliminary analysis with the atmospheric code CMFGEN, and we find that (despite the faint visual magnitudes) the bolometric luminosities of these stars are normal for early-type WNs. Our fitting suggests that these stars are evolved, with significantly enriched $\mathrm{N}$ and He. Their effective temperatures are also normal for early-type WNs. What is unusual about these stars is that they have a surprisingly small mass-loss rate compared to other early-type WNs. How these stars got to be the way they are (single star evolution? binary evolution?) remains an open question. For now, we are designating this class as WN3/O3, in analogy to the late-type WN "slash" stars.
\end{abstract}

Keywords. surveys, stars: Wolf-Rayet, stars: evolution, stars: early-type, galaxies: individual (LMC)

\section{A Modern Survey for Wolf-Rayet Stars in the Magellanic Clouds}

One of the long-standing tests of massive star evolutionary models is how well they do in predicting the observed ratios of various types of evolved massive stars as a function of metallicity (see, e.g., Maeder et al. 1980; Massey \& Johnson 1998; Meynet \& Maeder 2005; Meynet et al. 2007; Eldridge et al. 2008; Neugent et al. 2012a). Of these tests, possibly the most robust is that of comparing the relative number of WC- and WN-type Wolf-Rayet (WR) stars. Observationally, this quantity has long been known to be low in the Small Magellanic Cloud (SMC), higher in the Large Magellanic Cloud (LMC), and higher still in the Milky Way, in accord with the progression of metallicity. Vanbeveren \& Conti (1980) were the first to attribute this to the effects of metallicity on the mass-loss rates of main-sequence massive stars. However, as shown in many previous studies (e.g., Massey \& Conti 1983; Armandroff \& Massey 1985; Massey \& Johnson 1998; Neugent \& Massey 2011; Neugent et al. 2012a), the "observed" ratios of WCs to WNs can readily be biased towards stars of WC type, as the strongest lines in WCs are significantly $(\sim 10 \times)$ stronger than the strongest lines in WNs (Massey \& Johnson 1998). We have therefore begun new surveys for WRs in the star-forming galaxies of the Local Group (i.e., M33, 
Neugent \& Massey 2011, and M31, Neugent et al. 2012a) to allow more meaningful comparisons with the new generation of evolutionary models now becoming available (e.g., Eldridge et al. 2008; Ekström et al. 2012; Georgy et al. 2013).

As part of this process, our attention was drawn to the fact that the Geneva rotating models at LMC metallicity predict a WC to WN ratio of 0.09 (Neugent et al. 2012a) while the observed ratio is 0.23 according to the Breysacher et al. (1999) catalog (BAT99), as updated by Neugent et al. (2012b). Is this a problem with the single-star Geneva models, or could the problem be observational? For many years, our knowledge of the WR population of the Magellanic Clouds has been considered essentially complete. We realized that in the 15 years since the BAT99 catalog was published, there were seven new WRs found in the LMC, 6 of them of were of WN type. The other one was a rare WO star, found by ourselves (Neugent et al. 2012b). The WO star has very strong lines, and was found as part of a spectroscopic study of the stellar content of Lucke-Hodge 41, the home of S Doradus and many other massive stars. This discovery served as pretty much the last straw: we felt it behooved us to conduct a modern search for Wolf-Rayet stars in the Magellanic Clouds (Massey et al. 2014).

It was clear from the onset how we needed to do this: we would use the same sort of interference filters we had used so successfully to survey M33 and M31 for WRs (Neugent \& Massey 2011; Neugent et al. 2012a). We even knew where we wanted to do this: the Swope 1-m telescope had both a good image scale and a large field-ofview (FOV). Nevertheless, it would take about 800 fields and several hundred hours of observing to completely cover both Clouds. Fortunately they have just replaced the camera with one with an even larger FOV and much reduced overhead. Nevertheless this is a long term, multi-year project. In our first year (with 6 excellent nights) we were able to survey about $\sim 15 \%$ of each Cloud. Image-subtraction techniques then allowed us to identify stars which were brighter in a C III $\lambda 4650$ or a He II $\lambda 4686$ filter relative to neighboring continuum. We had concentrated on fields where WR stars were already known (following the philosophy attributed apocryphally to "Slick" Willie Sutton) and readily recovered all of the previously known WRs, except in the most crowded regions of the R136 cluster. We also re-discovered many previously known planetary nebulae and Of-type stars, both of which would have He II $\lambda 4686$ in emission. And, much to our relief, we also found a number of previously unknown WR candidates. Follow-up spectroscopy with Magellan allowed us to confirm 9 new WRs in the LMC in our first observing season. This increases the number of known WRs in the LMC by about $6 \%$, suggesting that the total WR population of the LMC has been underestimated by 10-40\%. While impressive, the greater significance is not in the quantity but the quality of what we found.

\section{What We Found}

Of the 9 newly found WRs in the LMC, 8 of these are WN stars. The other is another WO star. The WO is located only $9^{\prime \prime}$ from the one we found two years ago by accident in Lucke-Hodge 41 (Neugent et al. 2012b). Of the 8 WNs, 2 are normal WN3+mid-to-late O-type binaries; we were able to obtain two epochs of radial velocities that show the absorption and emission moving in anti-phase, as one would expect.

Five others (and possibly the sixth) are, however, quite peculiar. Naively we would classify their spectra as WN3+O3V. However, such a pairing would be unlikely for several reasons, the most unarguable one being that the absolute visual magnitudes of these six stars are quite faint, with $M_{V} \sim-2.3$ to -3.1 . This is much fainter than that of a typical O3 V star, with $M_{V} \sim-5.4$ (Conti 1988). Furthermore, we had two radial velocity epochs for three of these which failed to show variations. We will therefore refer to these 

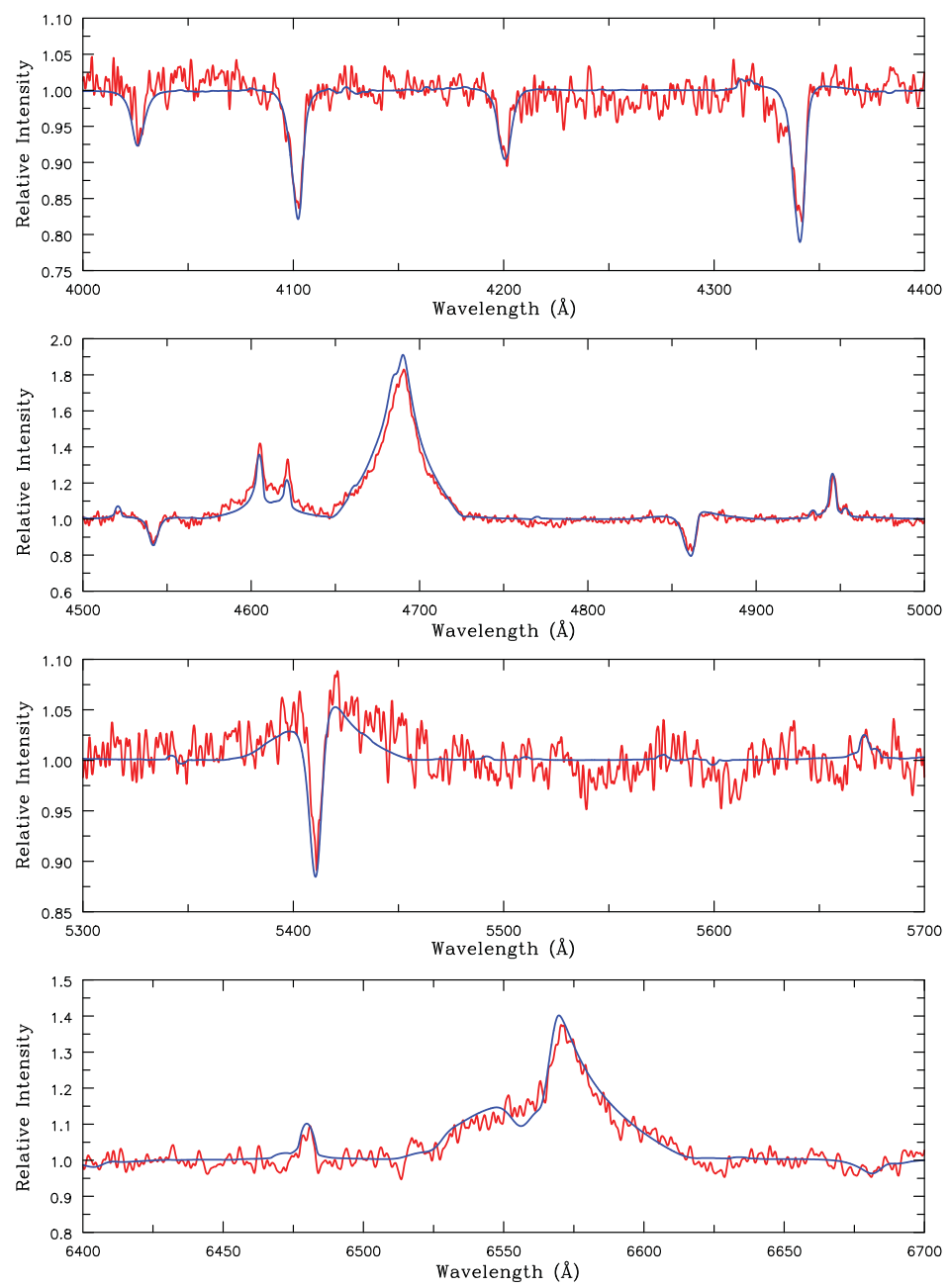

Figure 1. Our best CMFGEN fit to the optical spectrum of LMC170-2 From Massey et al. (2014) and used by permission.

stars as WN3/O3 WRs, with the slash reminiscent of the "transition" Ofpe/WN9 stars (Bohannan \& Walborn 1989; Bohannan 1990).

Could both the absorption and emission be coming from a single object? To explore this, we attempted to fit our optical spectrum of one of these stars (LMC170-2) with CMFGEN (Hillier \& Miller 1998), a stellar atmosphere code designed for hot stars with stellar winds where the usual assumptions of plane-parallel geometry and local thermodynamic equilibrium no longer hold. We found that we could obtain a very good fit with a single set of parameters as given in Table 1. Portions of the fit are shown in Fig. 1. A slightly different model also produced an adequate fit, and we give these parameters in Table 1 as well, to demonstrate the current uncertainties.

In Fig. 2 we show how these parameters compare to that of the LMC WN stars recently analyzed by Hainich et al. (2014). What we find is that the effective temperature and luminosity are normal for early-type WN stars (Fig. 2, left), but that the mass-loss rates are significantly lower (Fig. 2, right). In many ways this makes sense, as it suggests that the winds are weak enough that we see something like a normal stellar photosphere. 
Table 1. Parameters of our CMFGEN fits.

\begin{tabular}{lcc}
\hline Parameter & Best Fit & Pretty Good Fit \\
\hline Teff $(\mathrm{K})$ & 100,000 & 80,000 \\
$L / L \odot$ & $4 \times 10^{5}$ & $2.0 \times 10^{5}$ \\
$\dot{M}^{1}\left(M_{\odot} / \mathrm{yr}\right)$ & $1.2 \times 10^{-6}$ & $7.6 \times 10^{-7}$ \\
$\mathrm{He} / \mathrm{H}(\mathrm{by} \mathrm{\# )}$ & 1.0 & 0.5 \\
$\mathrm{~N}$ & $10.0 \times$ solar & $5.0 \times$ solar \\
$\mathrm{C}, \mathrm{O}$ & $0.05 \times$ solar & $0.05 \times$ solar \\
\hline
\end{tabular}

Notes:

${ }^{1}$ Assumes a clumping filling factor of 0.1 , wind terminal velocity of $v_{\infty}=2400 \mathrm{~km} / \mathrm{sec}$, and beta-type velocity law with $\beta=0.8$.
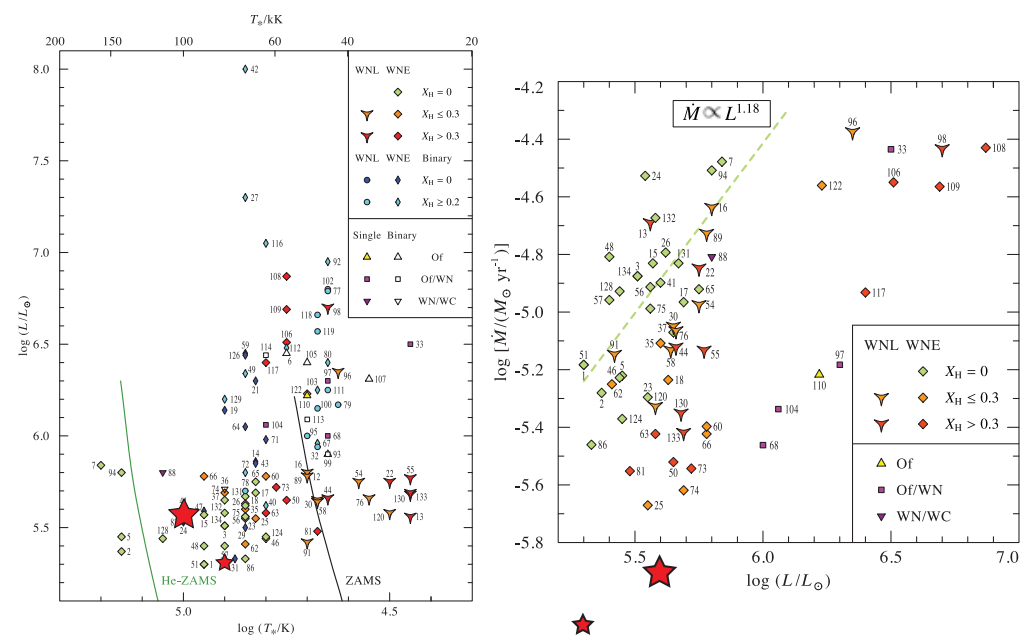

Figure 2. How the parameters of our fit of the WN3/O3 star LMC170-2 compare to those of other LMC WNs analyzed by Hainich et al. (2014). Our best fit is shown by a large star, while our "pretty good" fit is shown by a smaller star. Adapted from Figs. 6 and 7 of Hainich et al. (2014) and used by permission.

Of course, this now raises significant new questions: Why are the mass-loss rates low, and how did these stars evolve? Are binary models needed to produce such objects, or are these the hitherto unrecognized products of single-star evolution? If the former, then where is the spectroscopic signature of the companion?

We note that at this point the physical parameters are not well constrained (compare the two models in Table 1): the optical spectra lack multiple ionization stages of the same species, impacting our ability to constrain the effective temperature. We just learned that we were successful in obtaining time on HST to observe these objects in the UV; these spectra will provide data on additional ionization states (such as N IV $\lambda 1718$ ) as well as provide key diagnostics of the stellar wind, such as the C IV $\lambda 1550$ resonance line. We also plan to obtain higher $\mathrm{S} / \mathrm{N}$ optical data with Magellan, which should also help. We will continue to monitor these stars for radial velocity variations. We will be carrying out our survey for WRs in the rest of the Magellanic Clouds, and this will show us to see how many more of these WN3/O3s are out there.

And, of course, we do not know what other surprises await! In addition to these new WRs our study has also revealed two O8f?p stars, further examples of this rare class of magnetically-braked oblique rotators (Walborn et al. 2010). We have ten further nights 
scheduled on the Swope in the next Magellanic Cloud observing season (late 2014) with time for follow-up spectroscopy. So, stayed tuned!

This work has been supported by the National Science Foundation under AST-1008020 and by Lowell Observatory's research support fund, thanks to generous donations by Mr. Michael Beckage and Mr. Donald Trantow. D.J.H. acknowledges support from STScI theory grant HST-AR-12640.01. We appreciate the fine support we always receive at Las Campanas Observatory, where these observations were carried out. We are also grateful to our hosts here in Geneva for providing an opportunity to discuss this work in such a nice setting!

\section{References}

Armandroff, T. E. \& Massey, P. 1985, ApJ 291, 685

Bohannan, B. 1990, in C. D. Garmany (ed.), Properties of Hot Luminous Stars, Vol. 7 of Astronomical Society of the Pacific Conference Series, pp 39-43

Bohannan, B. \& Walborn, N. R. 1989, PASP 101, 520

Breysacher, J., Azzopardi, M., \& Testor, G. 1999, A \& AS 137, 117

Conti, P. S. 1988, NASA Special Publication 497, 119

Ekström, S., Georgy, C., Eggenberger, P., et al. 2012, A\& $A$ 537, A146

Eldridge, J. J., Izzard, R. G., \& Tout, C. A. 2008, MNRAS 384, 1109

Georgy, C., Ekström, S., Eggenberger, P., et al. 2013, A\& A 558, A103

Hainich, R., Rühling, U., Todt, H., et al. 2014, A\&\&A 565, A27

Hillier, D. J. \& Miller, D. L. 1998, ApJ 496, 407

Maeder, A., Lequeux, J., \& Azzopardi, M. 1980, A\&SA 90, L17

Massey, P. \& Conti, P. S. 1983, ApJ 273, 576

Massey, P. \& Johnson, O. 1998, ApJ 505, 793

Massey, P., Neugent, K. F., Morrell, N., \& Hillier, D. J. 2014, ApJ 788, 83

Meynet, G., Eggenberger, P., \& Maeder, A. 2007, in A. Vazdekis \& R. Peletier (eds.), Stellar Populations as Building Blocks of Galaxies, Vol. 241 of IAU Symposium, pp 13-22

Meynet, G. \& Maeder, A. 2005, A\&A 429, 581

Neugent, K. F. \& Massey, P. 2011, ApJ 733, 123

Neugent, K. F., Massey, P., \& Georgy, C. 2012a, ApJ 759, 11

Neugent, K. F., Massey, P., \& Morrell, N. 2012b, AJ 144, 162

Vanbeveren, D. \& Conti, P. S. 1980, A\&A 88, 230

Walborn, N. R., Sota, A., Maíz Apellániz, J., et al. 2010, ApJ (Letters) 711, L143

\section{Discussion}

SUNDQVIST: It seems to me that another interpretation of your two very interesting objects would be that they are just slightly evolved, still hydrogen-burning O-star with unusual high $T_{\text {eff }}$. Is this something you have considered?

MASSEY: It was our first thought - that maybe these were not evolved objects. However, our modeling has shown that $\mathrm{N}$ is strongly enhanced, and $\mathrm{C}$ and $\mathrm{O}$ are way down. The $\mathrm{He} / \mathrm{H}$ ratio is about 1 by number. I can't say what's going on in the core. But we have never seen an $\mathrm{O}$ star like this, and naively to me the $\mathrm{N}$ V and He II emission line strengths are similar to those of WN3 stars.

NAJARro: Phil, were you able to get a handle on $\log (g)$ (masses) for the objects?

MAssey: We adopted a $\log (g)$ of 5.0. That would lead to a mass of $15 M_{\odot}$, but the uncertainties are of order factors of 2 or 3 . John felt he could rule out a $\log (g)$ of 5.5 
for a temperature of $100000 \mathrm{~K}$, but it isn't well constrained. We hope with better optical data (higher S/N) and UV data with $H S T$ we can get more solid physical parameters.

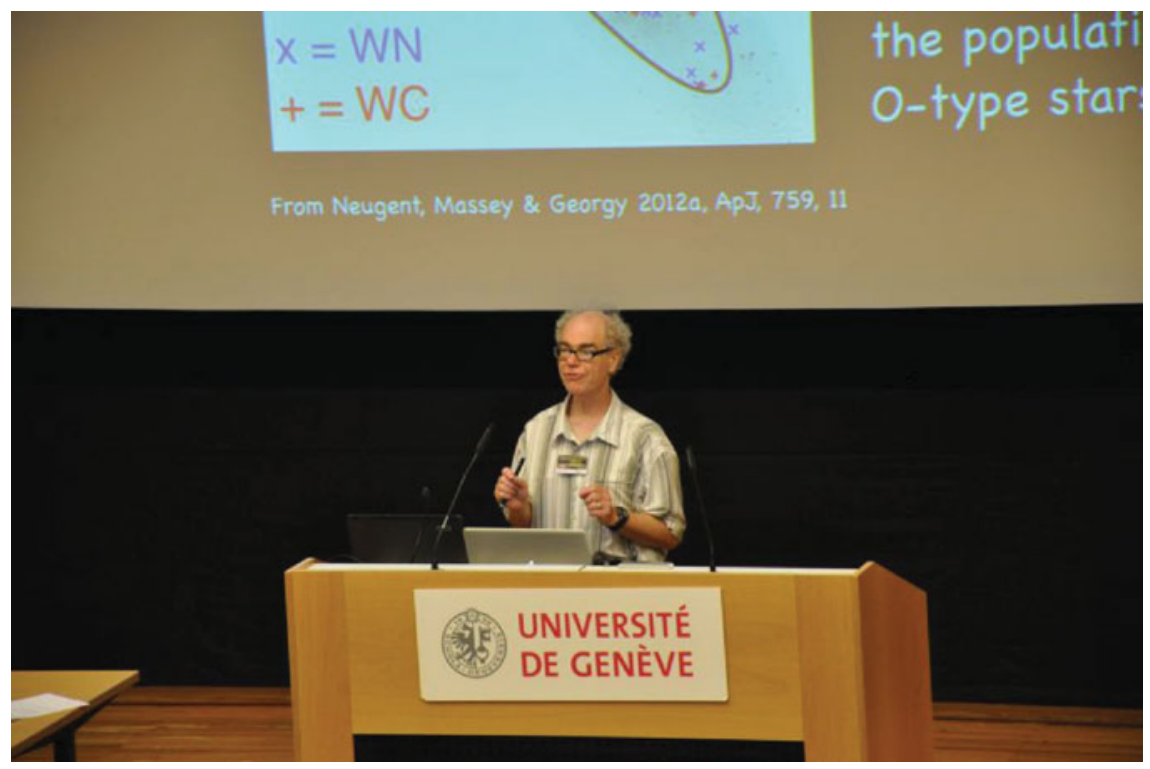

Philip Massey

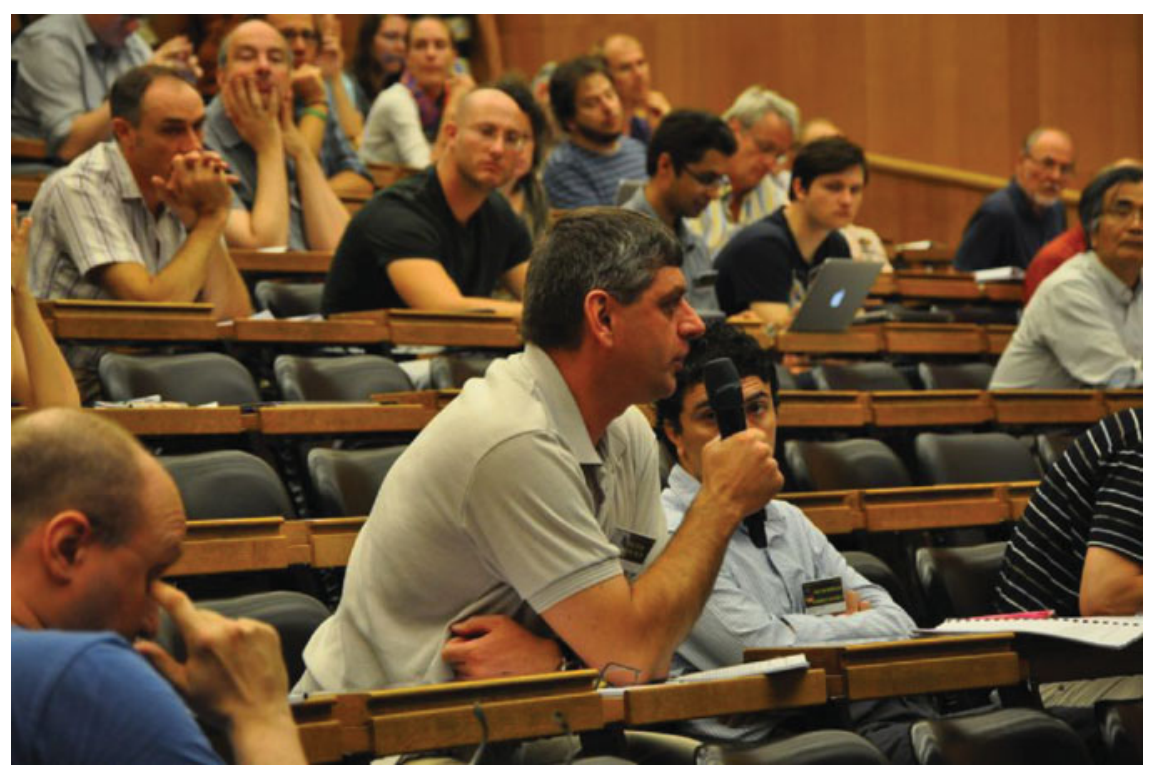

Victor Khalack asking a question 